\title{
Uncertainty area-based interference mitigation for cognitive radio
}

\author{
Mathieu Boutin ${ }^{1,2^{*}}$, Charles L Despins ${ }^{1,2,3}$ and Tayeb A Denidni ${ }^{1,2}$
}

\begin{abstract}
A new interference mitigation approach is presented for a Mobile Ad Hoc NETwork (MANET), acting as a secondary wireless network, which takes advantage of smart antennas and cognitive radio capabilities when arbitrarily deployed in the same area as a primary wireless network while both sharing the same spectrum. The MANET has to cope with the very coarse probabilistic estimate of the primary network antenna location, resulting from the absence of communication between both networks. Prior to MANET deployment, a Monte Carlo simulation is performed to evaluate the MANET's potential aggregate interference. Thus, both network managers have offline requirements to meet by such a simulation before approving their coexistence in the same environment. The adequate size of an uncertainty area surrounding each primary antenna, to respect those requirements, is therefore our main contribution to mitigate the MANET's interference. This is a novel solution for this kind of non-intrusive underlay spectrum sharing paradigm.
\end{abstract}

Keywords: cognitive radio, interference estimation, mobile ad hoc network, probabilistic location, radio coverage area, smart antennas, uncertainty area, underlay paradigm, wireless network coexistence

\section{Introduction}

The rapid growth of electromagnetic radio-spectrum use has made this natural resource a rather scarce asset for different wireless networks deployed in overlapping areas. Acute bandwidth shortage is predicted in the near future by considering the frequency bands already assigned for various applications such as television broadcast, cellular communications [1], etc. In contrast to this apparent spectrum scarcity has been the pervasive existence of spectrum opportunity as extensive measurements indicate that, at any given time and location, a large portion of licensed spectrum lies unused [2]. Even when a frequency band is actively used, the bursty nature of many applications results in abundant spectrum opportunities [3]. The relatively new spectrum underlay paradigm even allows simultaneous use of the shared spectrum by both the primary and secondary networks [4]. The cognitive radio concept is viewed as a promising way for improving the usage of this resource with two main objectives in mind: highly reliable

\footnotetext{
* Correspondence: mathieu.boutin@polymtl.ca

${ }^{1}$ INRS-EMT, Place Bonaventure, 800, de La Gauchetière West, Northwest portal, Suite 6900, Montreal, QC H5A 1K6, Canada

Full list of author information is available at the end of the article
}

communications whenever and wherever needed as well as efficient utilization of the radio spectrum [5].

The FCC has proposed an interference temperature model [6], for the underlay approach of wireless network coexistence, where unlicensed transceivers can operate on the same frequencies as licensed signals provided they can quantify and bound the resulting additional interference [4]. In this model, a secondary node (SN) senses the available spectrum band to further estimate the interference temperature experienced at each channel for each primary antenna (PA) and/or primary user that uses the channel. It is then allowed to transmit data on a channel provided the interference temperature for that channel does not exceed a predefined threshold at any aforementioned primary device [7]. Although temporarily abandoned by the FCC policy task force, the interference temperature model is still widely used among researchers as it provides an accurate measure at a receiving primary device for the acceptable level of RF interference in the spectrum band of interest [8]. The interference temperature of a channel is a function of the channel's average interference power for a given central frequency and spectral bandwidth. In this article though, an aggregate interference power is estimated in 
a radio agnostic fashion, using generic propagation loss parameters, so that no particular physical layer standards need to be specified. As such, we are concerned about the interference power instead of the temperature model, although the latter is indirectly considered by virtue of aggregate interference.

Many beamforming techniques may be used at transmitters and/or receivers belonging to the same network to enhance their communication. Choosing the best radiation beamwidth and direction at a given transceiver-so as to maximize the signal-to-interference-plusnoise ratio on desired links while minimizing it on any other link-is the challenging purpose of its smart antenna. This interference alleviation method also brought a lot of interest for the development of dynamic spectrum access strategies, where the transmitted power from SNs must be either lowered towards PAs or steered away from them, when sufficient information about PAs' location is available at SNs.

A major obstacle in estimating the aggregate interference at PAs can be the absence of communication between both networks to ensure non-intrusiveness, which can lead to a very inaccurate estimation of the PAs' location by the secondary network. The originality of this study is that our approach is especially designed to deal with this specific issue, which has rather been neglected in the past. Furthermore, it takes advantage of smart-antenna technology, but our scheme can be applied to any wireless network enabling such transmission directivity or not. The emphasis is also on the use of a Mobile Ad hoc NETwork (MANET) as the secondary network since MANETs will be soon very popular for a fast, easy, and lowcost deployment. Our original scheme also demonstrates that, by means of offline communicated coexistence requirements to be statistically satisfied by both networks, i.e., MANET interference underestimation and overestimation constraints imposed, respectively, by the primary and secondary networks, the unavailable online communication between both networks can be surprisingly counterweighted.

There will certainly be many future arising applications requiring the deployment of a MANET in the same environment as a primary network, from which any online communication is practically impossible and the knowledge of PAs' location by the MANET seriously lacks accuracy. Among many examples, one may think about a short-term special event occurring in a city and requiring mobile wireless video transfer for security purposes. Even though the precise PAs' location might be offline communicated to the such a MANET in this rather friendly situation, the radio-wave propagation behavior in this urban environment adds an non-negligible unpredictability factor. A military deployment in an enemy territory is another relevant example, although the assumption of offline communicated coexistence requirements may not hold (the primary network belonging to the enemy). Indeed, as the military MANET (intruder) wishes to reach a minimum QoS while not being detected by the enemy network, the previously mentioned interference estimation constraints still apply.

\section{Related work}

In many other approaches found in literature, pilot signals sent by a primary to a secondary network allow the latter to estimate the interference it causes to the former, so that interference constraints can only be violated within the desired limits as in [9], hence resulting on an intrusive secondary network deployment. Other authors as in [10-14] have proposed an alternative method-using omnidirectional antennas, beamforming technologies, or relay-assisted directional transmissionswith the drawback of having a constraint on the SNs position during the deployment, which is obviously not convenient when the secondary network is a MANET. A power control strategy serving as an alternative interference avoidance method has been presented in [15], which allows the secondary users to share the common spectrum used by primary users while not degrading the primary link's QoS, by not affecting its outage probability. However, this requires the secondary users to be able to frequently obtain the primary users' channel state information, which unfortunatly implies an online communication between them.

An interesting channel selection scheme under the interference temperature model has been presented in [16], where the interference is estimated in the frequency band of interest before SNs' transmission. However, SNs are assumed to know the exact waveform details and the modulation type of primary signal $a$ priori, so they are able to separate out the primary signals from other interfering signals because of noise and other SNs. The interference estimation accuracy is also highly dependent on SNs distribution over the deployment site. In [17], the proposition of a common control channel design, to be used by both the secondary and primary networks in order to exchange information, is not applicable in our non-intrusive MANET deployment scheme. By having the location information of the secondary as well as the primary users in [18], an overlaying cognitive ad hoc network can concurrently transmit peer-to-peer data in the presence of an infrastructuredbased connection in some region. However, it is still not applicable in our scheme because of our assumed PAs' location information inaccuracy for the MANET. The authors in [19] have aimed to maximize the throughput of SNs while keeping the interference temperature at 
PAs below a certain threshold, even without channel knowledge to the PAs. In fact, they study the optimal secondary-link beamforming pattern to steer interference away from PAs, which is unfortunatly too dependent on an accurate PAs' location information.

In this article, we now remove the need for the primary network to cater to the secondary network with a pilot signal and/or channel state information or details about the primary signal, so that the latter becomes totally non-intrusive, and also permits an arbitrary SNs deployment. To the best of our knowledge, nobody in the past has ever considered our main assumptions in the interference estimation of a secondary network towards a primary network: no online communication between both networks, coarse probabilistic estimate of PAs location obtained by the secondary network, and arbitrary deployment of the latter. For this reason, our simulation results presented in Section 5 cannot be compared with any other method from literature at this time, as our scheme is the first reference for this particular non-intrusive coexistence condition. We have already published some preliminary results of this work in [20], but this article now offers much more details on our proposed interference estimation technique, as well as a quite different and more efficient method to statistically justify the wireless coexistence approval. The following section explains how the results of a Monte Carlo simulation prior to the MANET deployment, provided that special coexistence requirements are communicated offline between both network managers, brings a novel interesting avenue for interference mitigation in this emerging open spectrum context.

\section{System models}

The communication model among SNs is exactly the same as reported in [21]. For the PAs, they are all assumed to have a unity gain and being omnidirectional for simplicity. In the remainder of this article, the set of all transmitting SNs is defined as $S_{T}$, and the set of all PAs is defined as $S_{P}$. For the worst case assessment, PAs are always considered to be in receiving mode. We assume all SNs to always know their exact location, by means of a GPS for instance. An SN's antenna beam is modeled as a circular sector since we assume the main lobe to be much more important than all the side lobes together as in [21].

Let $G_{T}(i)$ be the antenna gain of the $i$ th transmitting SN with its beamwidth $\theta(i)$. Similarly, let $G_{R}(j)$ be the antenna gain of the $j$ th receiving SN with its beamwidth $\theta(j)$. Let $d(i, p)$ be the distance between the $i$ th $\mathrm{SN}$ and $p$ th PA, and let $d_{0}$ be the far-field distance for the antenna of both types of transceiver. Our radio propagation model thus holds as long as $d(i, p) \geq d_{0}$, which is always respected in our simulations. We define $\alpha$ as the path loss exponent and $C(i, p)$ as a zero mean Lognormal random variable, with a standard deviation $\sigma_{C}$, representing the slow fading on the radio channel between $i$ and $p$ [22]. There is no need to consider fast fading since we assume it to be averaged in our model. This way of modeling the radio propagation is quite simple but does not have any impact on the usability of our interference estimation scheme explained subsequently. Indeed, any model could fit as long as the actual and estimated interference received at any PA are calculated accordingly.

The interference radius $r_{\mathrm{INT}}(i, p)$ is defined as the distance from $i$, transmitting with power $P_{T}(i)$, for which $p$ would receive the actual interference $I_{R}(i, p)$. If the antenna beamwidth and direction of the $i$ th $\mathrm{SN}$ are such that its coverage area overlaps the location of the $p$ th PA then we set $r_{\mathrm{INT}}(i, p)=d(i, p)$, otherwise we set $r_{\mathrm{INT}}$ $(i, p)=\infty$ for convenience. Let $r_{\mathrm{INT}, e}(i, p)$ be the MANET estimate of $r_{\mathrm{INT}}(i, p)$ which will be the subject of Section 4. $I_{R}(i, p)$ and its MANET estimate $I_{R, e}(i, p)$ are thus defined as

$$
\begin{gathered}
I_{R}(i, p) \equiv P_{T}(i) G_{T}(i)\left(r_{\mathrm{INT}}(i, p)^{-\alpha} C(i, p)\right) \\
I_{R, e}(i, p) \equiv P_{T}(i) G_{T}(i)\left(r_{\mathrm{INT}, e}(i, p)\right)^{-\alpha} .
\end{gathered}
$$

Because of the transmitted power attenuation as a function of distance from an $\mathrm{SN}$, the received power at any receiver ( $\mathrm{SN}$ or $\mathrm{PA}$ ) might be so small over a certain distance that it becomes irrelevant, even as interference. We thus define $P_{\mathrm{MNZ}}$ as the minimum non-zero power threshold below which any lower power is considered to be null. Let the distance from the $i$ th transmitting $\mathrm{SN}$, for its attenuated power to reach $P_{\mathrm{MNZ}}$ using $P_{T}(i)$ as its transmission power, be the radius $r_{M N Z}(i) \geq d_{0}$. By neglecting the random slow fading in this particular case for such small power, we obtain

$$
r_{\mathrm{MNZ}}(i) \equiv \sqrt[\alpha]{\frac{P_{T}(i) G_{T}(i)}{P_{\mathrm{MNZ}}}},
$$

which is obtained from an analogy to $I_{R}(i, p)$ in (1) with replacements:

$$
\begin{gathered}
I_{R}(i, p) \rightarrow P_{\mathrm{MNZ}}, \\
r_{\mathrm{INT}}(i, p) \rightarrow r_{\mathrm{MNZ}}(i), \\
C(i, p) \rightarrow 1 .
\end{gathered}
$$

By letting the $i$ th RCA be the radio coverage area of the $i$ th transmitting $\mathrm{SN}$, which is actually its transmission beam, $r_{\mathrm{MNZ}}(i)$ is in fact the radius of the circular sector representing it, such that the interference from $i$ is considered to be null outside its RCA.

A single SN of the MANET may generate interference to one or many PAs while transmitting towards other 
SNs. However, a PA cannot distinguish between individually received interference from transmitting $\mathrm{SNs}$, as it only perceives an aggregate interference denoted as $I_{A}$ $(p)$, and equivalently the MANET estimates of $I_{A}(p)$ is denoted as $I_{A, e}(p)$. As it becomes impossible for the MANET to exactly predict all radio propagation phenomena from SNs to PAs such as slow and fast fading, reflection, refraction, diffraction and so on [22], there is inevitably a difference or an error between $I_{A, e}(p)$ and $I_{A}(p)$ for all $p \in S_{P}$. In our interference mitigation approach explained subsequently in $3-\mathrm{B}$, this fact is rather an advantage so it is more appropriate to talk of an aggregate interference offset on $p$, denoted as $I_{A, o}(p)$, instead of an aggregate interference error, since this difference can probabilistically be selected by both the primary and secondary networks as explained subsequently. Those interference notations are thus defined as

$$
\begin{gathered}
I_{A}(p) \equiv \sum_{\forall h \in S_{T}} I_{R}(h, p), \\
I_{A, e}(p) \equiv \sum_{\forall h \in S_{T}} I_{R, e}(h, p), \\
I_{A, o}(p) \equiv I_{A, e}(p)-I_{A}(p) .
\end{gathered}
$$

\section{A. Probabilistic location of primary antennas}

Many techniques can be found in literature for a cognitive radio network to approximate the location of the primary network antennas. For example, the authors in [23] have combined the number of source estimations provided by the MUSIC [24] algorithm to the MC [25] algorithm to ensure cognitive radio's knowledge on the location of primary and secondary users. However, there are no perfect techniques which would always ensure a precise enough location estimate. Radio channel conditions may greatly vary over short periods of time, mutual interference among nodes may disturb adaptation processes, etc. We thus assume the MANET to never know the exact location of the PAs, but to rather have a coarse probabilistic estimate. Location estimates, such as the expectation, are much more useful if they are complemented with some indication about their precision. In order to visualize the uncertainty associated with the location of each PA, we assume that we have a probability distribution describing the uncertainty about the actual location. This can be done by drawing an Uncertainty Area (UA) centered at the estimated location such that the size and orientation describe the uncertainty of the location estimate as well as possible [26].

A bivariate Gaussian random variable with mean $\mu_{x}$ $(p)$ and $\mu_{y}(p)$, and standard deviation $\sigma_{x}(p)$ on the abscissa and $\sigma_{y}(p)$ on the ordinate, is used to model the
MANET location estimate of the $p$ th PA. This Gaussian distribution also has an inclination $\lambda(p)$ of any angle. By defining $\Omega_{\mathrm{UC}}(p)$ as the Uncertainty Coefficient, we thus obtain for the $p$ th PA an UA of elliptical shape centered at $\left(\mu_{x}(p), \mu_{y}(p)\right)$ with semimajor and semiminor axes, respectively, defined as

$$
\begin{aligned}
& A_{\mathrm{UC}}(p) \equiv \Omega_{\mathrm{UC}}(p) \sigma_{x}(p), \\
& B_{\mathrm{UC}}(p) \equiv \Omega_{\mathrm{UC}}(p) \sigma_{\gamma}(p) .
\end{aligned}
$$

As explained in the following section, $\Omega_{\mathrm{UC}}(p)$ is used to choose the size of the $p$ th UA, which is of key importance for the proposed interference mitigation scheme. A typical example of our non-intrusive underlay spectrum sharing context with an UA associated to every PA is presented in Figure 1, where it is shown that a PA might even be located outside of its UA if $\Omega_{\mathrm{UC}}(p)$ is not chosen sufficiently large.

\section{B. Requirements for coexistence}

Since we consider any online communication to be impossible between both networks, our scheme is based on the idea that, prior the MANET deployment in the same environment as the primary network, both of them must agree with the coexistence based on the results of an offline Monte Carlo simulation. This type of simulation method consists on independently repeating random experiments, from which statistical results are extracted. Those statistics are then used as a basis for decision-making regarding wireless coexistence. To this end though, a different coexistence requirement must be respected by each network, both related with $I_{A, o}(p) \forall p \in S_{P}$.

Indeed, what will prevent the coexistence acceptance by the primary network is a threatful aggregate interference underestimation (strictly negative $I_{A}, o(p)$ ) from the MANET obtained by simulation, which would make the latter erroneously believe that it can use an inappropriate transmission power for its communications once deployed. On the other hand, what will prevent the coexistence acceptance by the MANET is a too great aggregate interference overestimation (strictly positive $\left.I_{A}, O(p)\right)$ that would affect its QoS, by forbidding it from choosing necessary transmission power among its SNs, even if the received interference at PAs may be far from being critical.

For the primary network requirement and for all $p \in$ $S_{P}$, we define $I_{A, \mathrm{UM}}(p)$ as the aggregate interference Underestimation Margin of the $p$ th PA, with $\eta_{\mathrm{UM}}(p)$ as the maximum acceptable value of the probability $P_{\text {out }}$ $\mathrm{UM}(p)$ for an Underestimation Margin outage event $E_{\text {out,UM }}(p)$ to occur. The reason for not having $I_{A, \mathrm{UM}}$ $(p)$ and $\eta_{\mathrm{UM}}(p)$ as simply two constants, i.e. $I_{A, \mathrm{UM}}$ and $\eta_{\mathrm{UM}}$ applicable to any PA, is to be more general and 


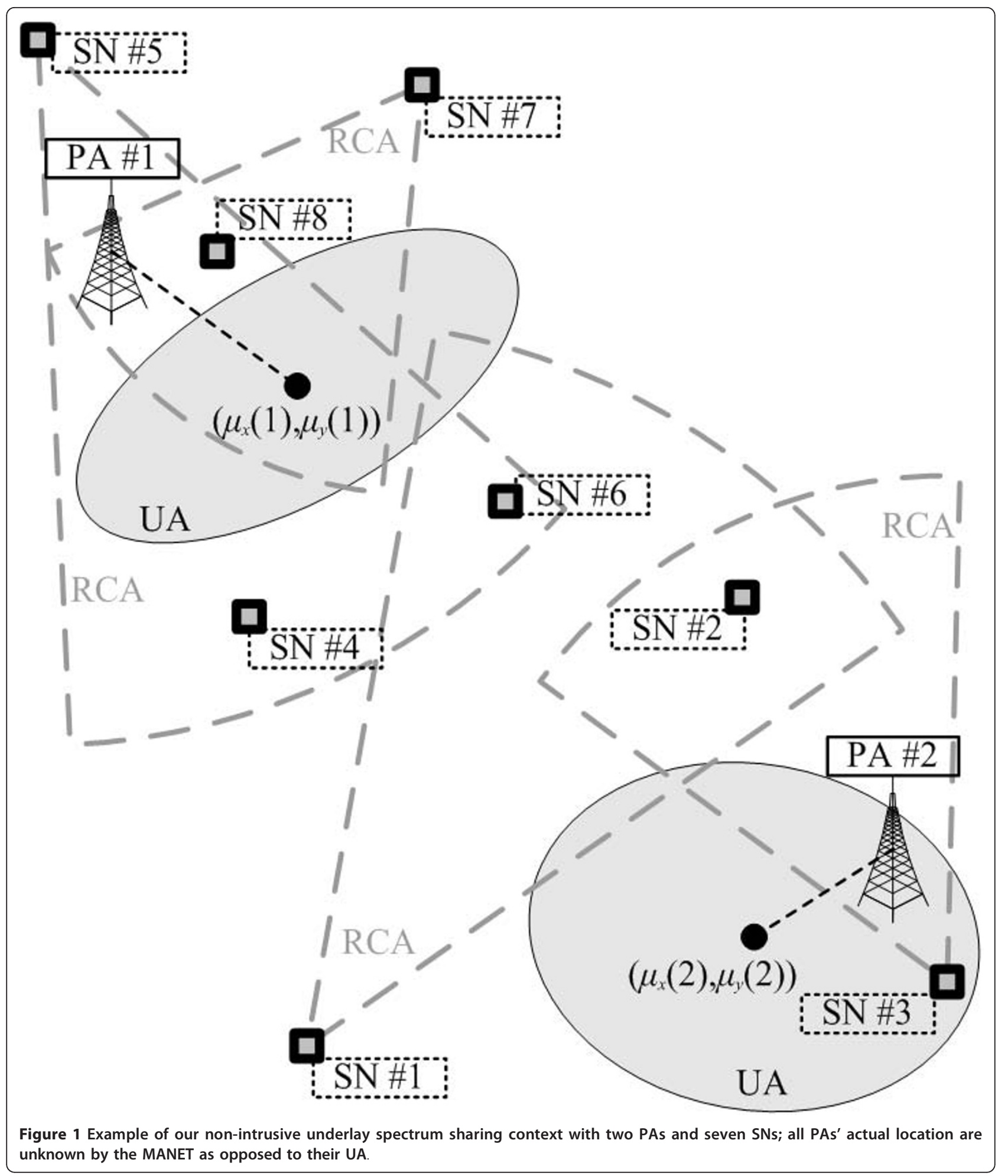

flexible. Indeed, the primary network might have a different interference constraint to be respected by each of its PAs. There are assorted examples of this possible differentiation necessity from a primary network, such as interference protection priority among PAs, type-specific traffic related to each $\mathrm{PA}$, variable reception gain of some PAs, etc. For the secondary network requirement, we define $I_{A \text {,OM }}$ as the aggregate interference overestimation margin of all PAs, with $\eta_{\mathrm{OM}}$ as the maximum acceptable value of the probability $P_{\text {out,OM }}(p)$ for an 
overestimation margin outage event $E_{\mathrm{out}, \mathrm{OM}}(p)$ to occur. Since the secondary network is not concerned with the interference overestimation with respect to a particular PA, but rather to any of them, there is no need to have a distinct value of $I_{A, \mathrm{OM}}$ and $\eta_{\mathrm{OM}}$ for each PA, like their counter parts $I_{A, \mathrm{UM}}(p)$ and $\eta_{\mathrm{UM}}(p)$ explained previously. These two coexistence requirements are thus summarized as follows for all $p \in S_{P}$ :

$$
\begin{gathered}
E_{\text {out }, \mathrm{UM}}(p) \equiv\left(I_{A, o}(p) \leq I_{A, \mathrm{UM}}(p)\right), \\
P_{\text {out, UM }}(p) \equiv \mathbb{P}\left(E_{\text {out, UM }}(p)\right) \leq \eta_{\mathrm{UM}}(p)
\end{gathered}
$$

and

$$
\begin{gathered}
E_{\text {out }, \mathrm{OM}}(p) \equiv\left(I_{A, o}(p) \leq I_{A, \mathrm{OM}}\right), \\
P_{\text {out }, \mathrm{OM}}(p) \equiv \mathbb{P}\left(E_{\text {out }, \mathrm{OM}}(p)\right) \leq \eta_{\mathrm{OM}} .
\end{gathered}
$$

By convention, each PA is considered separately, so that if any of both requirements is not respected by more than one PA, the number of outage events will simply equal the number of concerned PAs. For the $p$ th $\mathrm{PA}$, the events $E_{\mathrm{out}, \mathrm{UM}}(p)$ and $E_{\mathrm{out}, \mathrm{OM}}(p)$ are not jointly exhaustive since it is fortunately possible in a scenario that none of them occur. They are however mutually exclusive since both of them cannot occur simultaneously. They are not independent since, as they are both dependent on $\Omega_{\mathrm{UC}}(p)$, knowing that one of them occurs obviously affects the probability of the other to occur. In fact, as $\Omega_{\mathrm{UC}}(p)$ increases, $P_{\mathrm{out}, \mathrm{UM}}(p)$ tends to decrease while $P_{\text {out,OM }}(p)$ tends to increase, as explained in the next section and demonstrated by simulation in Section 5. All symbols presented in this section are finally summarized in Table 1, roughly ordered by appearance.

\section{New interference estimation technique}

The proposed non-intrusive interference mitigation approach, which we call Border-UA, intentionally tries to overestimate $I_{R}(i, p)$ for all $i \in S_{T}$ and $p \in S_{P}$ by calculating the estimated interference inside or at the edge of the UA surrounding $p$ 's mean location estimate, and the UA size of $p$ is chosen with the parameter $\Omega_{\mathrm{UC}}(p)$. If $i$ lies inside the UA of $p$, the MANET considers the interference that $i$ causes to the same geographical point as $i$ 's own location for $I_{R, e}(i, p)$ 's value by setting $r_{\text {INT, } e}$ $(i, p)=d_{0}$. On the other hand, if the $i$ th SN lies outside the UA of $p$, the MANET considers the interference that $i$ causes to the closest geographical point on the edge of this UA for $I_{R, e}(i, p)$ 's value. In the case where the RCA of $i$ does not intersect with the UA of $p$, then $I_{R, e}(i, p)$ is considered to be null by simply setting $r_{\mathrm{INT}, e}$

\begin{tabular}{|c|c|}
\hline$S_{T:}$ & Set of all transmitting SNs \\
\hline$S_{p:}:$ & Set of all PAs \\
\hline$i:$ & Any given transmitting $S N\left(i \in S_{T}\right)$ \\
\hline$j:$ & Any given receiving $\mathrm{SN}$ \\
\hline$p:$ & Any given $\mathrm{PA}\left(p \in S_{P}\right)$ \\
\hline$G_{T}(i):$ & i's antenna gain \\
\hline$G_{R}(j):$ & $j$ 's antenna gain \\
\hline$\theta(i) / \theta(j):$ & i's/j's beamwidth \\
\hline$d(i, p):$ & Distance between $i$ and $p$ \\
\hline$d_{0}:$ & Far-field distance, $d(i, p) \geq d_{0}$ \\
\hline$\alpha:$ & Path loss exponent \\
\hline$C(i, p):$ & Random slow fading between $i$ and $p$ \\
\hline$\sigma_{\subset}:$ & Slow fading standard deviation \\
\hline$P_{T}(i):$ & i's transmission power \\
\hline$r_{\mathbb{N} \mathrm{NT}}(i, p):$ & Interference radius from $i$ to $p$ \\
\hline$r_{\mathbb{I N T}, e}(i, p):$ & MANET estimate of $r_{\mathbb{I N T}}(i, p)$ \\
\hline$I_{R}(i, p):$ & Received interference from $i$ to $p$ \\
\hline$I_{R, e}(i, p):$ & MANET estimate of $I_{R}(i, p)$ \\
\hline$P_{\mathrm{MNZ}}:$ & Minimum non-zero power threshold \\
\hline$r_{M N Z}(i):$ & Distance from $i$ to reach $P_{M N Z}$ \\
\hline$I_{A}(p):$ & $p^{\prime}$ s aggregate interference \\
\hline$I_{A, e}(p):$ & MANET estimate of $I_{A}(p)$ \\
\hline$I_{A, O}(p):$ & $p^{\prime} s$ aggregate interference offset \\
\hline$\mu_{x}(p):$ & MANET estimate of $p$ 's mean $x$ location \\
\hline$\mu_{y}(p):$ & MANET estimate of $p^{\prime}$ s mean y location \\
\hline$\sigma_{x}(p):$ & MANET estimate of $p^{\prime}$ s std $x$ location \\
\hline$\sigma_{y}(p):$ & MANET estimate of $p^{\prime}$ s std y location \\
\hline$\lambda(p):$ & $p^{\prime}$ ' UA inclination \\
\hline$\Omega_{U C}(p):$ & $p^{\prime}$ s Uncertainty coefficient \\
\hline$A_{\cup C}(p):$ & $p^{\prime} s$ UA semimajor axis \\
\hline$B \cup C(p):$ & $p^{\prime} s$ UA semiminor axis \\
\hline$I_{A, U M}(p):$ & $I_{A}(p)^{\prime} s$ Underestimation margin \\
\hline$E_{\text {out,UM }}(p):$ & $I_{A, U M}(p)^{\prime}$ s outage event \\
\hline$P_{\text {out }, U M}(p):$ & Occurrence probability of $E_{\text {out,UM }}(p)$ \\
\hline$\eta_{\cup M}(p):$ & Max. acceptable value of $P_{\text {out,UM }}(p)$ \\
\hline$I_{A, O M}:$ & $I_{A}(S P)^{\prime} s$ Overestimation margin \\
\hline$E_{\text {out }, O M}(p):$ & $I_{A, \mathrm{OM}}$ 's outage event for $p$ \\
\hline$P_{\text {out }, \mathrm{OM}}(p):$ & Occurrence probability of $E_{\mathrm{out}, \mathrm{OM}}(p)$ \\
\hline$\eta_{\mathrm{OM}:}$ & Max. acceptable value of $P_{\text {out,OM }}\left(S_{P}\right)$ \\
\hline
\end{tabular}
$(i, p)=\infty$. An example of this simple but efficient technique is shown in Figure 2, entirely based on the example

\section{Table 1 Summary of model's symbols}

of Figure 1, where every $r_{\mathrm{INT}, e}(i, p)$ can also be compared with its counterpart $r_{\text {INT }}(i, p)$. The remainder of this section shows how to obtain $r_{\mathrm{INT}, e}(i, p)$ and, once those radii are known, the previously described methodology can be applied to generate the upcoming simulation of Section 5.

\section{A. RCA's intersection with UA}

The UA of the $p$ th PA is modeled as an ellipse rotated by an angle $\varphi_{E}$ and then translated at coordinates $\left(x_{E}\right.$, $y_{E}$ ), with a semimajor axis of $A_{E}$ and a semiminor axis 


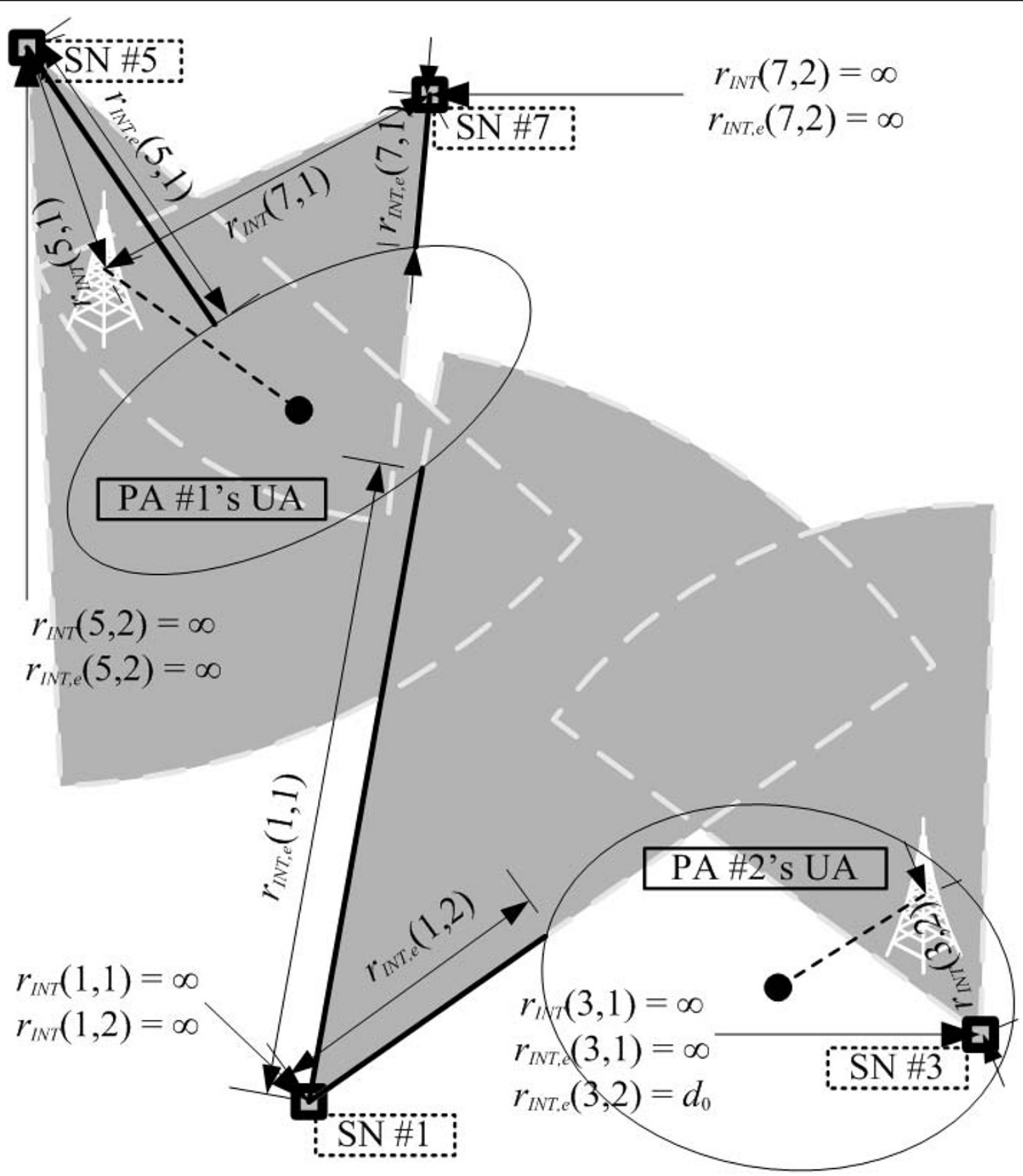

Figure 2 Illustration of our Border-UA interference estimation technique, taken from the example of Figure 1, showing $r_{\mathrm{INT}}(i, p)$ as well as $r_{\mathrm{INT}, e}(i, p)$ in order to respectively obtain $I_{R}(i, p)$ and $I_{R, e}(i, p), \forall i\left\lfloor S_{T}\right.$ and $\forall p L S_{p}$.

of $B_{E}$, which can be parametrically expressed as follows [27]:

$$
\begin{gathered}
x(t)=\left(\begin{array}{c}
x_{E}+A_{E} \cos \left(\varphi_{E}\right) \\
-B_{E} \sin \left(\varphi_{E}\right)
\end{array}\right), \\
y(t)=\left(\begin{array}{c}
y_{E}+B_{E} \sin (t) \cos \left(\varphi_{E}\right) \\
+A_{E} \cos (t) \sin \left(\varphi_{E}\right)
\end{array}\right), \\
A_{E} \geq B_{E}>0,-180^{\circ} \leq t \leq 180^{\circ} .
\end{gathered}
$$

One must be aware that the angle $t$ is relative to the positive semimajor axis and on the ellipse space, which is different from the 2D Euclidean space, not only by a translation and rotation (because of $x_{E}, y_{E}$ and $\varphi_{E}$ when different from zero), but also by a scale on both axes (because of $A_{E}$ and $B_{E}$ when different from one). For an external point to this ellipse with coordinates $\left(x_{P}, y_{P}\right)$, let $\vec{v}(t)$ be a vector starting at $\left(x_{P}, y_{P}\right)$ and ending at $(x(t)$, 
$y(t))$. We define $t_{P}$ as the angle of the external point on the ellipse space, which can be easily obtained. Let $\overrightarrow{v_{N}} \equiv \vec{v}\left(t_{N}\right)$ be the vector normal to the ellipse which occurs at $t=t_{N}$, and let $\overrightarrow{v_{T_{1}}} \equiv \vec{v}\left(t_{T_{1}}\right)$ and $\overrightarrow{v_{T_{2}}} \equiv \vec{v}\left(t_{T_{2}}\right)$ be the two vectors tangent to the ellipse which occur at $t=t_{T_{1}}$ and $t=t_{T_{2}}$, respectively. By extruding a circular sector of angle $\varphi_{L, R}$ from the external point, with vectors $\overrightarrow{u_{C}}, \overrightarrow{u_{R}}$, and $\overrightarrow{u_{L}}$ starting at $\left(x_{P}, y_{P}\right)$ and ending at the center, the right extremity, and the left extremity of the sector edge, respectively, the RCA of $i$ is modeled. We define the following angles on the Euclidean space:

$$
\begin{aligned}
\varphi_{N, T_{1}} & \equiv \angle\left(\overrightarrow{v_{N}}, \overrightarrow{v_{T_{1}}}\right), \\
\varphi_{N, T_{2}} & \equiv L\left(\overrightarrow{v_{N}}, \overrightarrow{v_{T_{2}}}\right), \\
\varphi_{R, N} & \equiv \angle\left(\overrightarrow{v_{R}}, \overrightarrow{v_{N}}\right), \\
\varphi_{C, N_{1}} & \equiv L\left(\overrightarrow{v_{C}}, \overrightarrow{v_{N_{1}}}\right), \\
\varphi_{L, N} & \equiv L\left(\overrightarrow{v_{L}}, \overrightarrow{v_{N}}\right) .
\end{aligned}
$$

Finally, in some circumstances (see Figure 3), we may need to use the vector $\vec{w} \equiv \overrightarrow{u_{L}}$ or $\vec{w} \equiv \overrightarrow{u_{R}}$, ending at $\left(x\left(t_{w}\right), y\left(t_{w}\right)\right)$, which occurs at $t=t_{w}$ (thus $\vec{w} \equiv \vec{v}\left(t_{w}\right)$ ). Such an UA and RCA with the aforementioned parameters are depicted in Figure 4.

The radius $r$ of the RCA can be obtained by four different ways depending on the circumstances as shown by the flow diagram in Figure 3, which actually gives the value of $r_{\mathrm{INT}, e}(i, p)$. Indeed, the model described above can be directly applied to our MANET and primary network with the following replacements:

$$
\begin{gathered}
\left(x_{E}, y_{E}\right) \rightarrow\left(\mu_{x}(p), \mu_{y}(p)\right), \\
A_{E} \rightarrow A_{\mathrm{UC}}(p), \\
B_{E} \rightarrow B_{\mathrm{UC}}(p), \\
\left(x_{p}, \gamma_{p}\right) \rightarrow(x(i), y(i)), \\
\varphi_{L, R} \rightarrow \theta(i), \\
r \rightarrow r_{\mathrm{INT}, e}(i, p) .
\end{gathered}
$$

Nonetheless, the parameters $t_{N}, t_{T_{1}}, t_{T_{2}}$ and $t_{w}$ need to be approximated in order to do so, which is explained next.

\section{B. Iterative methods for parameters approximation}

A vector starting at $\left(x_{P}, y_{P}\right)$ and ending at $(x(t), y(t))$ perpendicular to the UA would result on having its length minimized. This is actually the case with $\overrightarrow{v_{N}}$ for which $\|\vec{v}(t)\|$ has its global minimum at $t=t_{N}$. Because of the non-linearity of $\|\vec{v}(t)\|$, it becomes much more affordable to approximate $t_{N}$ by an iterative method rather than trying to get its exact value analytically [28]. Using the BFGS Quasi-Newton method with a mixed quadratic and cubic line search procedure [29], and starting this unconstrained nonlinear optimization with $t_{P}$ as the initial estimate, we attempt to find the minimum of $\|\vec{v}(t)\|$ to obtain an approximation of $t_{N}$. It has to be noted that, depending on the circumstances, i.e., the location of the external point with respect to the UA as well as the value of the ellipse parameters, it may occur for $\|\vec{v}(t)\|$ to have two minima. For the one-minimum case, the convergence is guaranteed from the fact that it is impossible for $t_{P}$ to be located at the unique maximum. For the two-minima case, it is also impossible for $t_{P}$ to be located at one of both maxima, and will always be located on the side of both maxima leading to the global minimum, hence the convergence is also guaranteed.

For two vectors starting at $\left(x_{P}, y_{P}\right)$ and ending tangent to the UA on both sides of $\overrightarrow{v_{N}}$, two minima must be found for the function

$$
f(t)=\frac{-\overrightarrow{v_{N}} \cdot \vec{v}(t)}{\left\|\overrightarrow{v_{N}}\right\|\|\vec{v}(t)\|}
$$

occurring at $t=t_{T_{1}}$ and $t=t_{T_{2}}$. The function in (11) is actually the negative of the cosine of the angle between $\overrightarrow{v_{N}}$ and $\vec{v}(t)$, from the vector dot product equation, where we have added the negative sign in order to find two minima instead of two maxima. Since one of its maxima is located at $t=t_{N}$ which has previously been approximated, both minima are obviously located on both sides of it. Using the same iterative method as the one to approximate $t_{N}$, we now find the two minima of $f(t)$ with $t_{N} \pm 1^{\circ}$ as initial estimates. For the input parameters used in our simulation, the choice of these initial estimates ensures that both executions of the search procedure will always distinctly converge to both minima. However, the value of the initial estimates to guarantee convergence will depend on the typical scenario to consider as well as the required precision, but this minor issue is rather irrelevant with today's computing capabilities and precision.

An iterative approach is also required to approximate $t_{w}$. For this purpose, we use an optimization algorithm based on a golden section search and parabolic interpolation [30] to find the minimum of the function

$$
g(t)=\frac{-\vec{w} \cdot \vec{v}(t)}{\|\vec{w}\|\|\vec{v}(t)\|}
$$

between bounds $b_{1}$ and $b_{2}$ (see Figure 3), which are used to prevent the algorithm from getting the wrong minimum, so that the convergence to the global minimum is guaranteed. Similarly as for $f(t)$ in (11) described previously, the function $g(t)$ in (12) is the negative of the 


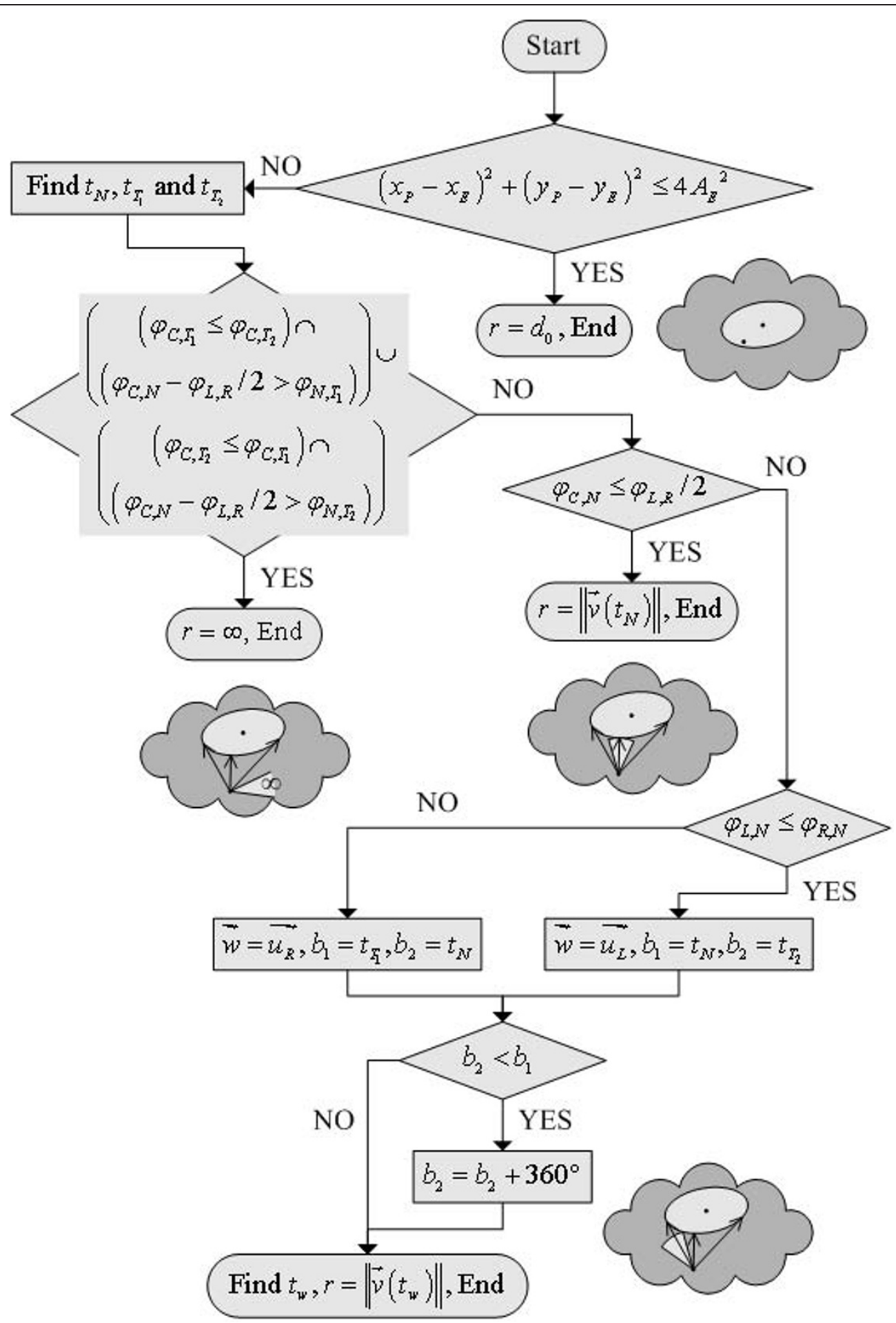

Figure 3 Flow diagram of the algorithm to set the RCA's radius $r$.

cosine of the angle between $\vec{w}$ and $\vec{v}(t)$ from the vector dot product equation.

The presented approaches to approximate $t_{N}, t_{T_{1}}, t_{T_{2}}$, and $t_{w}$ are all based on the search of a global minimum of a one-parameter nonlinear function, for which the initial estimate is judiciously chosen to guarantee convergence and correctness. However, one must be aware that the complexity and precision are directly related to 


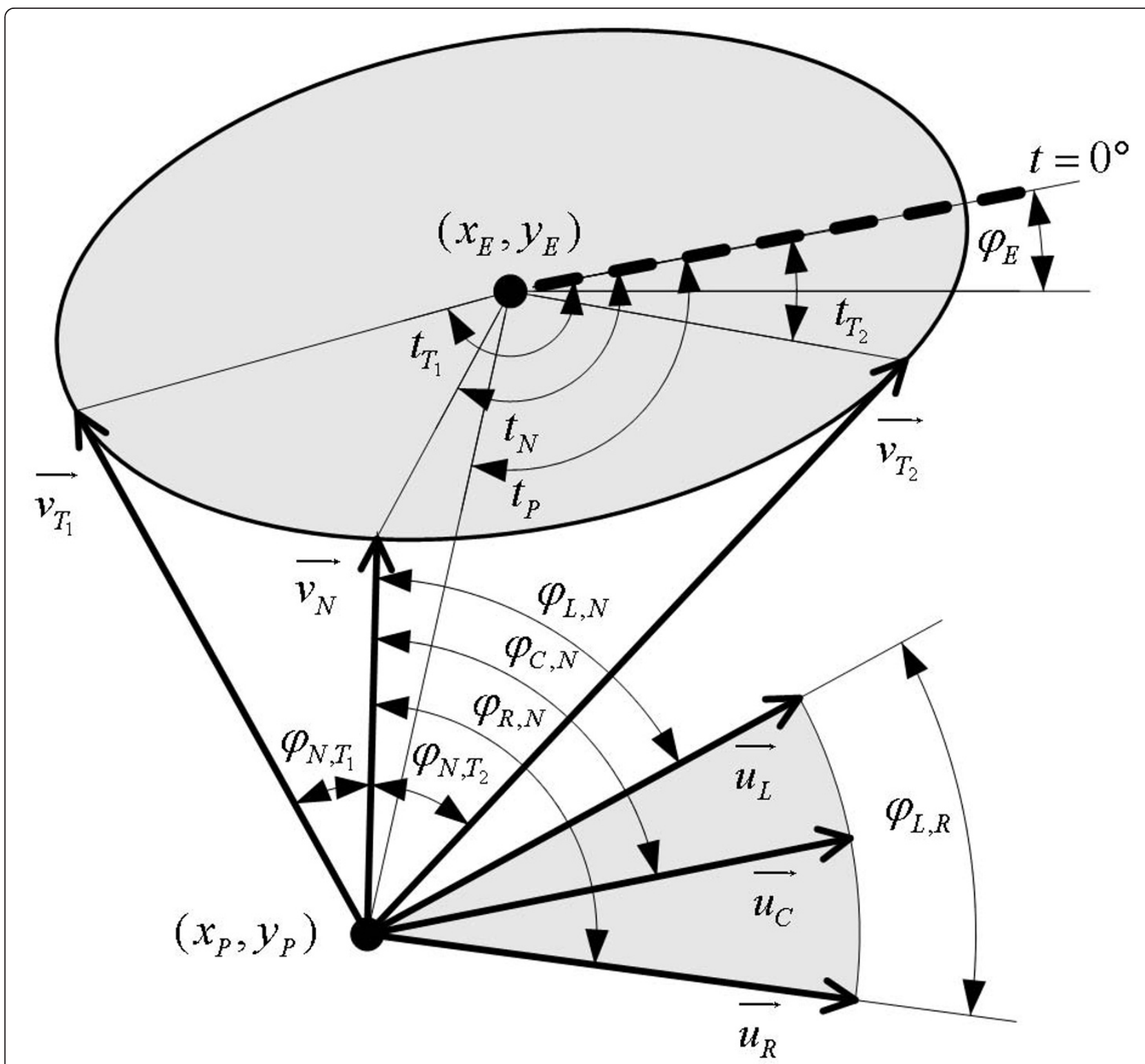

Figure 4 Visual representation of an SN's transmission RCA with respect to the UA of a PA.

the termination tolerance on both $t$ and the function value, and the allowed maximum number of both iterations and function evaluations of the aforementioned iterative algorithms. Fortunately, because of the random nature of radio-wave propagation, the exactness of these values is not crucial, since a not so small difference between the exact and approximated values is very unlikely to influence the interference estimation.

\section{Simulation}

A Monte Carlo simulation with Matlab was performed to assess our Border-UA technique. The simulation is made of a large number of independently generated scenarios forming the samples from which relevant statistics are extracted. For each scenario, SNs as well as the estimated location of only one PA, i.e., the mean $\left(\mu_{x}\right.$ $\left.(p), \mu_{y}(p)\right)$, are randomly uniformly distributed on a $2 \mathrm{D}$ simulation area. A bivariate Gaussian distribution with means $\mu_{x}(p)$ and $\mu_{y}(p)$, and standard deviations $\sigma_{x}(p)$ and $\sigma_{y}(p)$ is then used for the actual location (in meters) of the unique PA, followed by an uniformly distributed inclination with $\left(\mu_{x}(p), \mu_{y}(p)\right)$ as the rotation center. The resultant PAs actual location may lie outside the simulation area, but is still considered. Since we consider the worst case situation where all PAs are in receiving mode, the presence of one PA does not affect 
SNs or other PAs in any way, so all PAs can thus be treated independently and separately. Therefore, setting more than one PA per scenario would be useless as the obtained statistics from only one PA are the same for all other PAs. The primary network sets $I_{A, \mathrm{UM}}\left(S_{P}\right)$ with $\eta_{\mathrm{UM}}\left(S_{P}\right)$ while the MANET sets $I_{A \text {, OM }}$ with $\eta_{\mathrm{OM}}$ as their respective coexistence requirements.

For each scenario, $I_{A, o}(p)$ is calculated for each possible value of $\Omega_{\mathrm{UC}}(p)$ from a chosen relevant continuous interval. This results on a large amount of $I_{A, o}(p)$ values associated with each $\Omega_{\mathrm{UC}}(p)$, forming an Empirical Cumulative Distribution Function (ECDF). Such an ECDF is then separated into a partial ECDF for all $I_{A, o}$ $(p)<0$, and a partial Empirical Complementary CDF (ECCDF) for all $I_{A, o}(p)>0$. The former is used by the primary network to assess the aggregate interference underestimation, whereas the latter is used by the MANET to assess the overestimation. It has to be noted that neither of both partial CDFs includes $I_{A, o}(p)=0$ since it only occurs when no RCA intersects the UA of $p$ and actual location, so it is not of any help. The upcoming example represents a typical possible simulation from which the primary and secondary networks could justify their coexistence decision. One should note that, because of the uniqueness of our non-intrusive coexistence condition still not considered in literature up to now, our simulation results cannot be compared with any previous method, as already mentioned.

\section{A. Results of a coexistence example}

We consider a $1.0 \mathrm{~km}^{2}$ deployment site as a coexistence example, where the primary network is composed of 3 PAs respectively named "PA \#1", "PA \#2", and "PA \#3" for $S_{P}=\{1,2,3\}$, and the MANET is composed of 35 SNs. We use our previously developed Closest Successful Receiver to Random Transmitters (CSRRT) algorithm presented in [21] to establish the communication links among SNs in order to obtain $S_{T}$. The simple but realistic resultant connectivity from this algorithm depends on the constant receiving SN noise power $N_{R}$, the SINR threshold $\gamma_{\text {SINR }}$, the link factor $\xi$, and the same constant narrow beamwidth used by both transmitting and receiving SNs (ensuring a relatively high connectivity). All simulation input parameters are listed in Table 2.

Those parameters are chosen so as to represent a MANET deployment with high node density, in a typical urban area. Such a cluttered RF environment gives rise to the relatively high imprecision on PAs location estimate by the MANET. In regard to the coexistence requirements, different $I_{A, \mathrm{UM}}(p)$ 's and $\eta_{\mathrm{UM}}(p)$ 's value were attributed for each PA, with the objective of providing a better idea of the flexibility offered by our scheme. Meanwhile, $I_{A \text {,OM}}$ 's and $\eta_{\mathrm{OM}}$ 's values were
Table 2 Simulation input parameters

\begin{tabular}{l} 
Scenarios: \\
Area $=1.0 \times 1.0 \mathrm{~km}^{2}$ \\
Number of PAs $=3$ \\
Number of $\mathrm{SNS}=35$ \\
Number of scenarios $=25000$ \\
$\sigma_{x}(p) \sim \sigma_{y}(p) \cdot \cup[1,4], \forall p \in S_{P}$ \\
$\sigma_{y}(p) \sim[6,18], \forall p \in S_{P}$ \\
$\Omega_{\mathrm{UC}}(p) \in[0.0,0.2, \ldots, 5.0], \forall p \in S_{P}$ \\
Radio propagation: \\
$\alpha=2.1$ \\
$\sigma_{C}=0.2$ \\
$d_{0}=1.0 \mathrm{~m}$ \\
$P_{\mathrm{MNZ}}=10^{-15} \mathrm{~W}$ \\
Coexistence requirements \\
Primary network \\
$I_{A, \mathrm{UM}}(1)=-10^{-6} \mathrm{~W}, \eta_{\mathrm{UM}}(1)=0.02$ \\
$I_{A, \mathrm{UM}}(2)=-5 \times 10^{-6} \mathrm{~W}, \eta_{\mathrm{UM}}(2)=0.008$ \\
$I_{A, \mathrm{UM}}(3)=-10^{-5} \mathrm{~W}, \eta_{\mathrm{UM}}(3)=0.001$ \\
Secondary network \\
$I_{A, \mathrm{OM}}=5 \times 10^{-5} \mathrm{~W}, \eta_{\mathrm{OM}}=0.15$ \\
$\mathrm{CSRRT}$ algorithm \\
$N_{R}=5 \times 10^{-15} \mathrm{~W}$ \\
$\gamma_{\mathrm{SINR}}=13 \mathrm{~dB}$ \\
$\xi=1.25$ \\
$\theta(i)=\theta(j)=60^{\circ}$ \\
$G_{T}(i)=2 \pi / \theta(i), g_{R}(j)=2 \pi / \theta(j)$ \\
\hline
\end{tabular}

simply set as if the MANET were not too demanding with respect to its own performance-for its deployment to be possible.

All simulation results are shown in Figures 5, 6, and 7. The five-number summaries of the random variable $I_{A, o}$ $(p)$ for the underestimation and overestimation are presented in Figure 5a,b, respectively. This statistical summary, as a function of $\Omega_{\mathrm{UC}}(p)$, comprises the minimum value, the first quartile, the median, the third quartile and the maximum value. Still as a function of $\Omega_{\mathrm{UC}}(p)$, Figure $5 \mathrm{c}$ shows the occurrence probability of having an aggregate interference underestimation $\left(I_{A, o}(p)<0\right)$, overestimation $\left(I_{A, o}(p)>0\right)$ or a null offset $\left(I_{A, o}(p)=\right.$ $0)$. For each scenario and a given value of $\Omega_{\mathrm{UC}}(p)$, exactly and only one of those three aforementioned possibilities occurs, since the simulation is actually performed with only one PA as explained previously.

It is important to note that, as shown in Figure 5c, the number of available samples (scenarios) is not constant as a function of $\Omega_{\mathrm{UC}}(p)$ in Figure 5a,b. When $\Omega_{\mathrm{UC}}(p)$ $=0.0$, the underestimation and overestimation occurrence probabilities are about the same because $p$ does not have an UA yet, and the null offset probability is at its maximum (although quite low) for the same reason. Therefore, as $\Omega_{\mathrm{UC}}(p)$ increases, the number of 

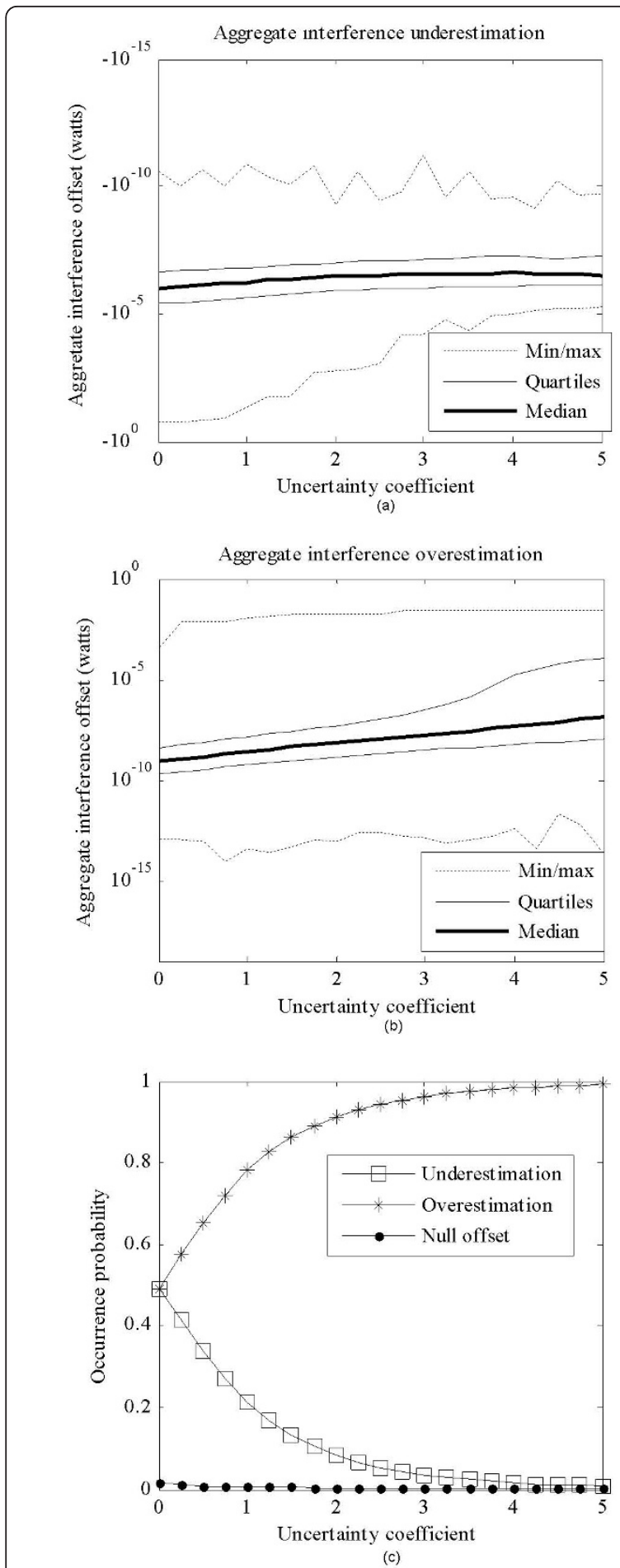

Figure 5 Statistics of $I_{A \prime o}(p)$ from simulation: (a) five-number summary of underestimation, (b) five-number summary of overestimation, (c) occurrence probabilities. underestimation samples for Figure 5a decreases while the number of overestimation samples for Figure $5 \mathrm{~b}$ increases. The five-number summaries of Figure $5 \mathrm{a}, \mathrm{b}$ finally show that $I_{A, o}(p)$ clearly tends to statistically increase as $\Omega_{\mathrm{UC}}(p)$ increases, which is obviously expected.

The partial ECDF for the aggregate interference underestimation is presented in Figure 6a where the thick dashed lines represent the requirements of the primary network to be respected $\left(I_{A, \mathrm{UM}}\left(S_{P}\right)\right.$ and $\left.\eta_{\mathrm{UM}}\left(S_{p}\right)\right)$. The partial ECCDF for the aggregate interference overestimation is presented in Figure $6 \mathrm{~b}$, where the thick dashed lines represent the requirement of the MANET to be respected ( $I_{A, \mathrm{OM}}$ and $\left.\eta_{\mathrm{OM}}\right)$. Figure $7 \mathrm{a}$ presents $P_{\text {out,UM }}\left(S_{P}\right)$ as a function of $\Omega_{\mathrm{UC}}\left(S_{P}\right)$, where the thick dashed lines show the minimum value of $\Omega_{\mathrm{UC}}\left(S_{P}\right)$ for the primary network requirements to be respected. Figure $7 \mathrm{~b}$ presents $P_{\text {out,OM }}\left(S_{P}\right)$ as a function of $\Omega_{\mathrm{UC}}\left(S_{P}\right)$ where the black thick dashed lines show the maximum value of $\Omega_{\mathrm{UC}}\left(S_{P}\right)$ for the MANET requirement to be respected. One can also see from Figure $7 \mathrm{~b}$ the gray thick dashed lines at the minimum value of $\Omega_{\mathrm{UC}}\left(S_{P}\right)$ taken from Figure 7a, so the interval of possible value of $\Omega_{\mathrm{UC}}\left(S_{P}\right)$ can be clearly seen for each PA in order for the coexistence to be accepted by both networks.

\section{B. Discussion}

Both Figures 6 and 7 are essential in the coexistence decision process after the Monte Carlo simulation has been completed. Notwithstanding the fact that Figure 7 is by far the most convenient between both figures to precisely determine the permitted $\Omega_{\mathrm{UC}}\left(S_{P}\right)$ 's interval granted that $I_{A, \mathrm{UM}}\left(S_{p}\right), \eta_{\mathrm{UM}}\left(S_{P}\right), I_{A, \mathrm{OM}}$ and $\eta_{\mathrm{OM}}$ are fixed, Figure 6 gives a much better overview of the worthy value range of those coexistence requirement parameters. Indeed, an informal decision can easily be taken by both network managers by only taking a glance at Figure 6, which is not possible with Figure 7 as a degree of liberty is lost since $I_{A, \mathrm{UM}}\left(S_{p}\right)$ and $I_{A, \mathrm{OM}}$ are now implicit to the curves. It is clearly shown in Figure $7 \mathrm{~b}$ that the coexistence is possible if $\Omega_{\mathrm{UC}}(1) \in[2.19$, 3.25] for "PA \#1", $\Omega_{\mathrm{UC}}(2) \in[0.9,3.25]$ for "PA \#2" and $\Omega_{\mathrm{UC}}(3) \in[2.75,3.25]$ for "PA \#3". The optimal value for $\Omega_{\mathrm{UC}}\left(S_{P}\right)$ to choose among those intervals is however out of the scope of this article as it may depend on many factors. For instance, it could be settled that the primary network remains always privileged by choosing the maximum permitted value of $\Omega_{\mathrm{UC}}\left(S_{P}\right)$ so that its aggregate interference is minimized. Under other circumstances, some performance metrics could be 


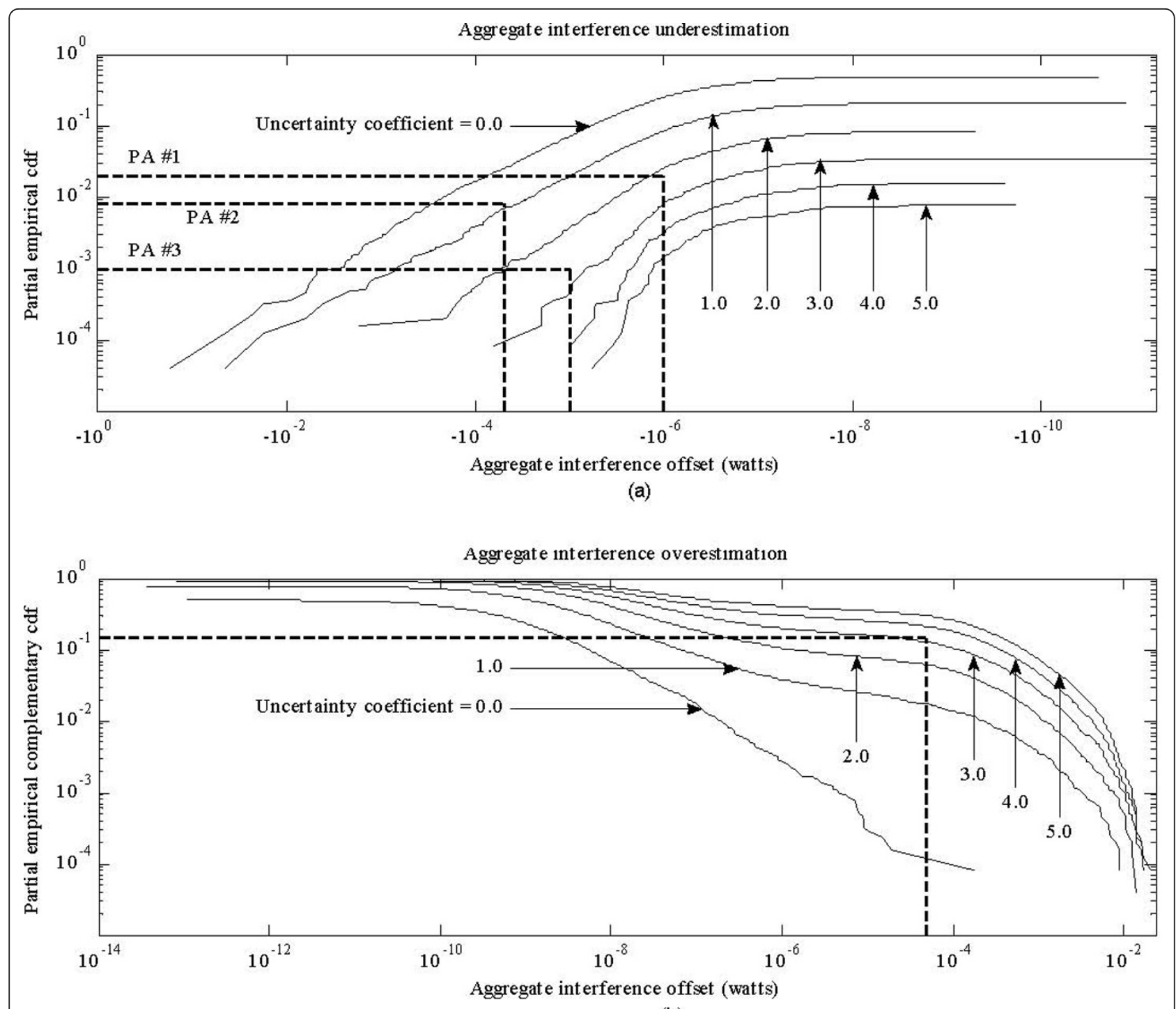

(b)

Figure 6 Simulation results: (a) partial ECDF for the aggregate interference underestimation and showing the primary network requirements $\left(I_{A, \cup M}\left(S_{P}\right)\right.$ and $\left.\eta_{\cup M}\left(S_{P}\right)\right)$, (b) partial ECCDF for the aggregate interference overestimation and showing the MANET requirements $\left(I_{A, O M}\right.$ and $\left.\eta_{\mathrm{OM}}\right)$.

accounted by both networks so that the value of $\Omega_{\mathrm{UC}}$ $\left(S_{P}\right)$ is chosen such that an agreed Pareto optimum is obtained. Choosing the optimal $\Omega_{\mathrm{UC}}\left(S_{P}\right)$ might be the subject of our future research.

One should note that the presented simulation results strongly depend on our antenna and radio propagation models, the simulation area, the number of PAs and SNs as well as the algorithm used (CRSST) to establish communication links among SNs. Changing any of those attributes could give results at a rather different scale, but the main trends would always be the same. Even if modeling smart-antenna radiation-beam patterns with circular sectors might seem too simplistic, real beam patterns can actually be precisely modeled by many adjacent circular sectors, so our scheme is thus fully applicable for any model to reach the desired realworld fidelity.

\section{Conclusion}

A novel and very promising interference mitigation approach has been described in this article, which greatly takes advantage of the use of smart antennas by secondary network nodes when arbitrarily deployed in the same area as primary network antennas. Our scheme is especially designed to cope with the very poor or non-existent online communication between 


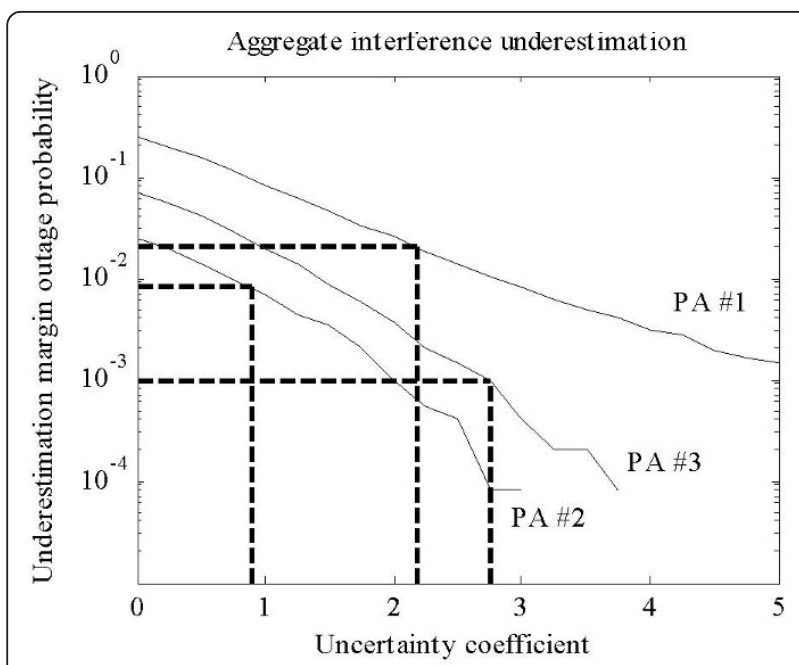

(a)

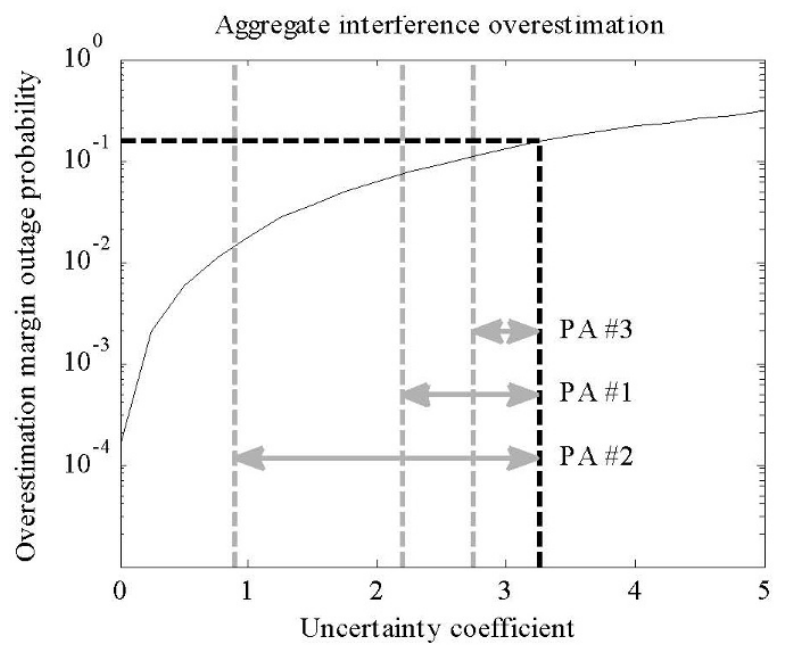

(b)

Figure 7 Simulation results: (a) $P_{\text {out }, U M}\left(S_{P}\right)$ for the primary network decision on the minimal permitted value of $\Omega_{U \mathrm{C}}\left(S_{P}\right)$; (b) $P_{\text {out,OM }}\left(S_{P}\right)$ for the MANET decision on the maximal permitted value of $\Omega_{\mathrm{UC}}\left(S_{P}\right)$.

primary and secondary networks, which is highly prone to lead to an inaccurate probabilistic location estimate of each PA by the SNs. By selecting the appropriate size of an UA surrounding the estimated location of each PA, the trade-off between aggregate interference underestimation and overestimation can statistically be tuned by a Monte Carlo simulation prior the secondary network deployment, provided that wireless coexistence requirements are communicated offline between both networks.

Ensuring non-intrusive integration of a MANET in the same spatial and spectral environment as a primary network, by assuming no online communication between them, undoubtedly makes any coexistence adaptation quite tedious by both parties. Without any feedback from the concurrent network, the other one will not be able to reach its quasi-optimal performance, and vice versa. Nevertheless, if each network keeps a history of its activity and QoS-related metrics over time, a periodic offline feedback is still possible between both network managers, and coexistence requirements updated accordingly. Such an offline adaptation might be really valuable in the situations explained in [31] where malicious radio nodes (as enemies from a third network, for instance) try to spoof the online communicated feedback between the secondary and primary networks, in order for the intelligent adaptive capabilities of the former to unfortunately play against itself.

Our scheme could even be employed without any requirement communicated offline between networks, as for military applications. The secondary network requirement would then become a QoS constraint, and the primary network requirement would become an interference constraint (for the secondary network not to be detected due to its own transmissions). Determining the optimal $\Omega_{\mathrm{UC}}(p)$, instead of an acceptable interval, might even be done by online adaptation in our future study, for which many original approaches are yet to be discovered. Using the same coexistence constraints as mentioned in this article, but by providing even greater values for $\Omega_{\mathrm{UC}}\left(S_{P}\right)$ 's upper and lower bounds, is of real interest and also part of our future study. On the one hand, the greater is the UAs size of a PA for fixed coexistence constraints, the greater is the probability of this PA to be located inside of its $\mathrm{UA}$, and hence a better interference protection is permitted. On the other hand, if one assumes the primary network to be also composed of primary users receiving data from these PAs, as for the upcoming IEEE 802.22 WRAN standard for the digital TV Broadcast spectrum sharing [32], these primary users will also benefit from a better interference protection if their PAs use a greater UAs size.

\section{Author details}

${ }^{1}$ INRS-EMT, Place Bonaventure, 800, de La Gauchetière West, Northwest portal, Suite 6900, Montreal, QC H5A 1K6, Canada ${ }^{2}$ LRTCS-UQAT, 450, $3^{\text {rd }}$ Avenue, Suite 103, Val-d'Or, QC J9P 1S2, Canada ${ }^{3}$ Prompt Inc., 1155, University Street, Suite 903, Montreal, QC H3B 3A7, Canada

\section{Competing interests}

The authors declare that they have no competing interests.

Received: 28 February 2011 Accepted: 21 November 2011 Published: 21 November 2011

\section{References}

1. M Thoppian, S Venkatesan, R Prakash, R Chandrasekaran, MAC-layer scheduling in cognitive radio based multi-hop wireless networks, in IEEE Proceedings of the 2006 International Symposium on World of Wireless, Mobile and Multimedia Network, 191-202 (2006) 
2. M McHenry, Spectrum white space measurements, Presented to New America Foundation Broadband Forum; Measurements by Shared Spectrum Company. (June 2003)

3. Q Zhao, L Tong, A Swami, Y Chen, Decentralized cognitive MAC for opportunistic spectrum access in ad hoc networks: a POMDP framework. IEEE J Sel Areas Commun. 25(3), 589-600 (2007)

4. T Clancy, Formalizing the interference temperature model. Wiley J Wirel Commun Mob Comput. 7(9), 1077-1086 (2007). doi:10.1002/wcm.482

5. S Haykin, Cognitive radio: brain-empowered wireless communications. IEEE J Sel Areas Commun (invited paper). 23(2), 201-220 (2005)

6. FCC Notice of Inquiry and Notice of Proposed Rule Making, In the matter of establishment of an interference temperature metric to quantify and manage interference and to expand available unlicensed operation in certain fixed, mobile and satellite frequency bands, Et Docket No. 03-237 (November 13, 2003)

7. M Sharma, A Sahoo, KD Nayak, Channel modeling based on interference temperature in underlay cognitive wireless networks, in IEEE International Symposium on Wireless Communication Systems (ISWCS), Reykjavik, Iceland, 224-228 (October 21-24, 2008)

8. T Chen, H Zhang, M Kartz, CogMesh: cognitive wireless mesh networks, in Proceedings of IEEE Globecom 2008 (Workshop on Wireless Mesh and Sensor Networks), New Orleans, LA, USA, (November 30-December 4, 2008)

9. DI Kim, LB Le, E Hossain, Joint rate and power allocation for cognitive radios in dynamic spectrum access environment. IEEE Trans Wirel Commun. 7(12), 5517-5527 (2008)

10. $S$ Huang, $Z$ Ding, $X$ Liu, Non-intrusive cognitive radio networks based on smart antenna technology, in IEEE Global Telecommunications Conference (GLOBECOM), Washington, DC, USA, 4862-4867 (November 26-30, 2007)

11. MF Hanif, PJ Smith, On the statistics of cognitive radio capacity in shadowing and fast fading environments. IEEE Trans Wirel Commun. 9(2), 844-852 (2010)

12. S Yiu, M Vu, V Tarokh, Interference and noise reduction by beamforming in cognitive networks. IEEE Trans Commun. 57(10), 3144-3153 (2009)

13. G Zhao, J Ma, GY Li, T Wu, YH Kwon, A Soong, C-Y Yang, Spatial spectrum holes for cognitive radio with relay-assisted directional transmission. IEEE Trans Wirel Commun. 8(10), 5270-5279 (2009)

14. $M$ Vu, N Devroye, $V$ Tarokh, On the primary exclusive region of cognitive networks. IEEE Trans Wirel Commun. 8(7), 3380-3385 (2009)

15. Y Chen, G Yu, Z Zhang, H Chen, P Qiu, On cognitive radio networks with opportunistic power control strategies in fading channels. IEEE Trans Wirel Commun. 7(7), 2752-2761 (2008)

16. M Sharma, A Sahoo, KD Nayak, Channel selection under interference temperature model in multi-hop cognitive mesh networks, in IEEE 2nd International symposium on New Frontiers in Dynamic Spectrum Access Networks, Dublin, Ireland, 133-136 (April 17-20, 2007)

17. KR Chowdhury, IF Akyildiz, OFDM-based common control channel design for cognitive radio ad hoc networks. IEEE Trans Mob Comput. 10(2), 228-238 (2011)

18. L-C Wang, A Chen, Effects of location awareness on concurrent transmissions for cognitive ad hoc networks overlaying infrastructure-based systems. IEEE Trans Mob Comput. 8(5), 577-589 (2009)

19. YJ Zhang, AM-C So, Optimal spectrum sharing in MIMO cognitive radio networks via semidefinite programming. IEEE J Sel Areas Commun. 29(2), 362-373 (2011)

20. M Boutin, C Despins, T Denidni, Interference protection on legacy devices with cognitive radio using smart antennas, in IEEE 70th Vehicular Technology Conference, Anchorage, Alaska, USA, (September 20-23, 2009)

21. M Boutin, C Despins, T Denidni, S Affes, A novel interference safety margin for cognitive radio MANET using smart antennas. Special Issue on Wireless Communication on Underground and Confined Areas. J Commun. 4(4), 257-266 (2009)

22. M Boutin, A Benzakour, CL Despins, S Affes, Radio wave characterization and modeling in underground mine tunnels. IEEE Trans Antenn Propag. 56(2), 540-549 (2008)

23. O Duval, A Punchihewa, F Gagnon, C Despins, VK Bhargava, Blind multisources detection and localization for cognitive radio, in IEEE Global Telecommunications Conference (GLOBECOM), New Orleans, LA, USA, (November 30-December 4, 2008)

24. LC Godara, Smart Antennas (CRC Press, 2004)

25. A Punchihewa, OA Dobre, S Rajan, R Inkol, Cyclostationarity-based algorithm for blind recognition of OFDM and single carrier linear digital modulations, in IEEE 18th International Symposium on Personal, Indoor and Mobile Radio Communications (PIMRC), Athens, Greece, (September 3-

7, 2007)

26. P Kontkanen, P Myllymäki, T Roos, H Terri, K Valtonen, $H$ Wettig, Topics in probabilistic location estimation in wireless networks, in IEEE 15th International Symposium on Personal, Indoor and Mobile Radio Communications (PIMRC), vol. 2. Barcelona, Spain, 1052-1056 (September 5-8, 2004)

27. CD Miller, ML Lial, DI Schneider, Fundamentals of College Algebra, 3rd edn (Scott Foresman/Little, 1990)

28. SJ Ahn, W Rauh, M Recknagel, Ellipse fitting and parameter assessment of circular object targets for robot vision, in, in IEEE/RSJ Proceedings, International Conference on Intelligent Robots and Systems (IROS), vol. 1. Kyongju, South Korea, 525-530 (October 17-21, 1999)

29. R Fletcher, Practical Methods of Optimization, Vol 1 Unconstrained Optimization (Wiley, 1980)

30. RP Brent, Algorithms for Minimization Without Derivatives (Prentice-Hall, Englewood Cliffs, NJ, 1973)

31. TC Clancy, N Goergen, Security in cognitive radio networks: threats and mitigation, in IEEE 3rd International Conference on Cognitive Radio Oriented Wireless Networks and Communications (CrownCom), Singapore, Singapore, (May 15-17, 2008)

32. C Stevenson, G Chouinard, L Zhongding, H Wendong, S Shellhammer, W Caldwell, IEEE 802.22: the first cognitive radio wireless regional area network standard. IEEE Commun Mag. 47(1), 130-138 (2009)

doi:10.1186/1687-1499-2011-175

Cite this article as: Boutin et al:: Uncertainty area-based interference mitigation for cognitive radio. EURASIP Journal on Wireless Communications and Networking 2011 2011:175.

\section{Submit your manuscript to a SpringerOpen ${ }^{\mathcal{O}}$ journal and benefit from:}

- Convenient online submission

- Rigorous peer review

- Immediate publication on acceptance

- Open access: articles freely available online

- High visibility within the field

Retaining the copyright to your article

Submit your next manuscript at $>$ springeropen.com 